\title{
CERN Proton Synchrotron booster space charge simulations with a realistic model for alignment and field errors*
}

\author{
V. Forte \\ CERN, Meyrin, CH-1211 Geneva, Switzerland-Université Blaise Pascal, Clermont-Ferrand, France
}

\author{
E. Benedetto and M. McAteer
}

CERN, Meyrin, CH-1211 Geneva, Switzerland

(Received 8 October 2015; revised manuscript received 3 November 2016; published 28 December 2016)

The CERN Proton Synchrotron booster (PSB) is one of the machines of the LHC injector chain which will be upgraded within the LHC Injectors Upgrade (LIU) project. The injection energy of the PSB will be increased to $160 \mathrm{MeV}$ in order to mitigate direct space charge effects, considered to be the main performance limitation, aiming to double the brightness for the LHC beams. In order to better predict the gain to be expected, space charge simulations are being carried out. As a first step, benchmarking between simulations and measurements is needed. Efforts to establish a realistic modeling of field and alignment errors aim at extending the basic model of the machine toward a more realistic one. Simulations of beam dynamics with strong space charge and realistic errors are presented and analyzed in this paper.

DOI: 10.1103/PhysRevAccelBeams.19.124202

\section{INTRODUCTION}

The CERN Proton Synchrotron booster (PSB), being the first synchrotron of the LHC chain, is in a process of being upgraded to accomplish the LHC Injectors Upgrade project (LIU) requests and to double the brightness of the beams to the LHC. A new injection energy at $160 \mathrm{MeV}$ (instead of the actual $50 \mathrm{MeV}$ ) from the Linac4 is foreseen to mitigate the space charge (SC) effects, which constitute the main concern in terms of beam quality. The evaluation of future space charge effects on the beam has to be performed through simulation codes. To benchmark the code with the measurements, an accurate model of the PSB lattice is necessary, including elements misalignments and magnetic field errors. To underline the importance of a complete model in combination with space charge effects, measurements and simulations are shown in this paper on a $160 \mathrm{MeV}$ flat plateau.

Based on measurements, the lattice errors are determined. With this improved model, two specific cases concerning a half-integer $\left(2 Q_{y}=9\right)$ and an integer $\left(Q_{y}=4\right)$ resonance are analyzed. These resonances are of particular importance in the machine operations.

\section{ESTIMATION OF LATTICE ERRORS}

The distribution of focusing errors in the machine lattice was estimated using the linear optics from closed orbits

\footnotetext{
†vincenzo.forte@cern.ch

Published by the American Physical Society under the terms of the Creative Commons Attribution 3.0 License. Further distribution of this work must maintain attribution to the author $(s)$ and the published article's title, journal citation, and DOI.
}

(LOCO) method [1]. The change to the closed orbit in response to the 26 dipole orbit correctors ( 13 in each plane) and to momentum offsets was measured, and then nonlinear least squares fitting was used to find the set of model parameters that minimizes the difference between the measured and model orbit response:

$$
\begin{aligned}
& \chi^{2}=\sum_{k=i, j} R_{k}^{2}=\sum_{i, j}\left[\left(\frac{\partial z_{i}}{\partial \theta_{j}}\right)_{\text {model }}-\left(\frac{\partial z_{i}}{\partial \theta_{j}}\right)_{\text {meas }}\right]^{2} \cdot \frac{1}{\sigma_{i j}^{2}}
\end{aligned}
$$

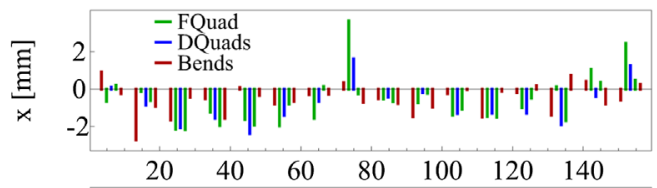

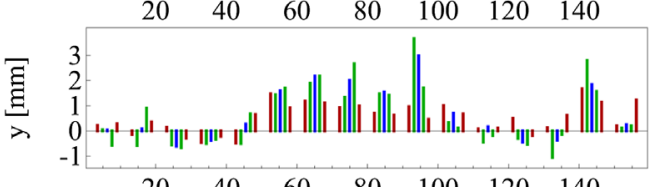

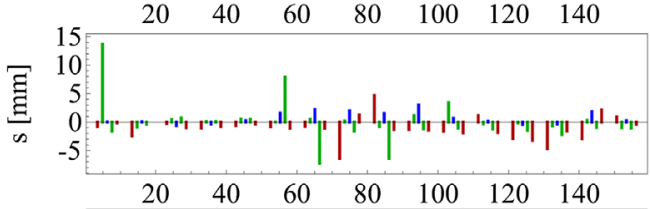

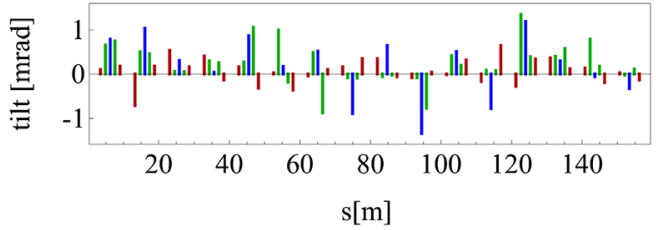

FIG. 1. Transverse and longitudinal alignment errors and tilts of triplet quadrupoles and bending magnets in Ring 2, from May 2013 tunnel survey. 


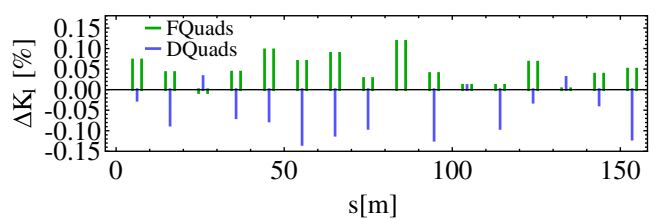

FIG. 2. Relative errors (percent) of triplet quadrupole magnet strengths in Ring 2, determined by LOCO analysis.

where $i$ is the index of the beam-position monitors (BPMs), $j$ is the index of the orbit correctors, $z$ represents both the horizontal and vertical orbit positions, $\theta$ is the bending angle and $\sigma_{i j}$ is the standard error of the linear fit [2]. The measured orbit response to opposite-plane dipole magnets was small compared with the uncertainty of the measurement, so only the diagonal portions of the orbit response matrix were used for the LOCO fitting. The model parameters used as variables were the strengths of each of 16 defocusing quads and each of the 16 pairs of focusing quads (with the pair of focusing magnets in each triplet treated as a single variable to reduce degeneracy problems in the fitting), and the calibration of the dipole correctors and of the BPMs. The lattice
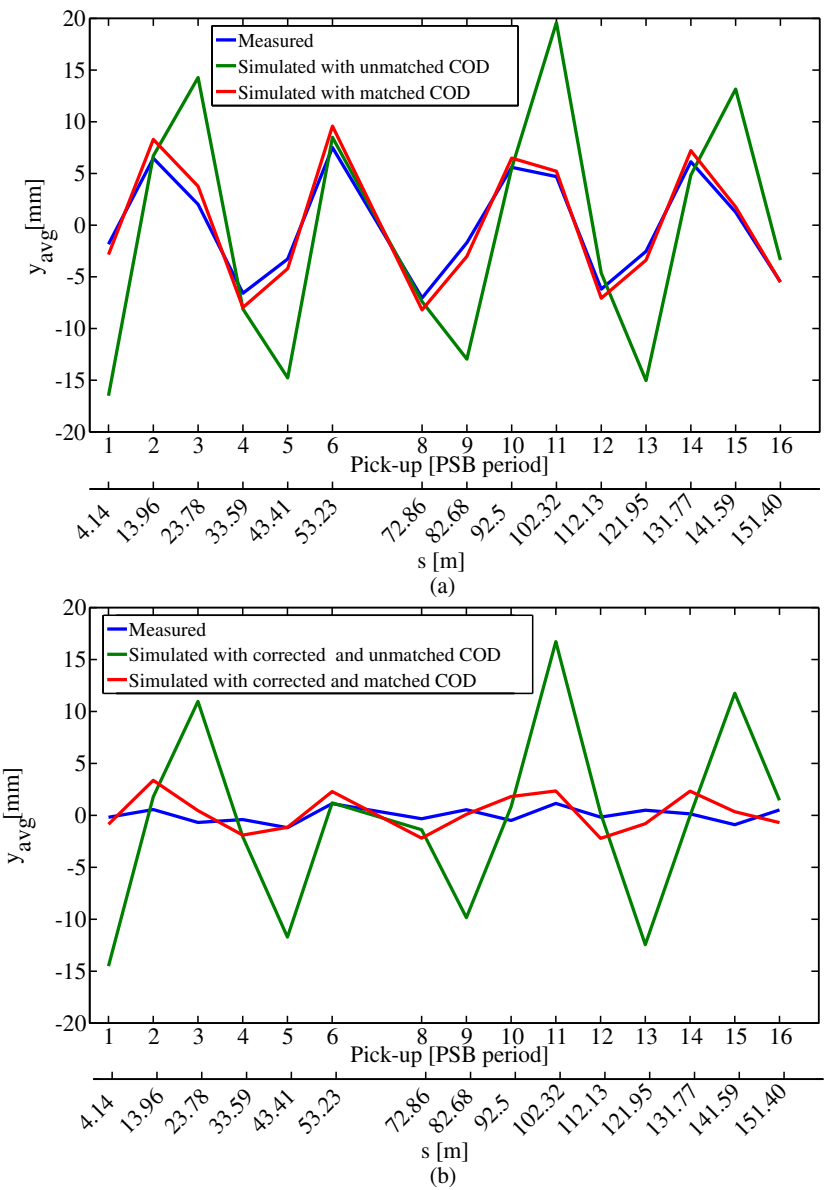

FIG. 3. The COD without (a) and with (b) measured COD matching for the lowest $\left(Q_{y}=4.09\right)$ vertical working point. model used for the LOCO fitting included alignment errors (Fig. 1) for the triplet quads, bending magnets, and $\mathrm{BPM} /$ multipole corrector package stacks as measured in a May 2013 tunnel survey [3]. The focusing errors (Fig. 2), found from a LOCO fitting for the working point of $\left(Q_{x}, Q_{y}\right)=(4.20,4.26)$, were on the order of one per mil of the nominal integrated gradients of the triplet magnets [4].

For the simulations near the integer resonance, where closed orbit distortions (COD) had a large effect on beam loss patterns, the orbit of the model was matched to the measured orbit. A set of steering errors which minimized the difference between the measured and model closed orbit was added to the thirteen orbit corrector dipoles in the lattice. Figure 3 shows the measured and simulated vertical COD for $Q_{y}=4.09$ (bottom). Without steering corrections, the model predicts a much larger COD than what is observed. But with steering corrections the model reproduces the measured orbit fairly well at all working points, both without [Fig. 3(a)] and with [Fig. 3(b)] orbit correctors active in the real machine.

\section{THE HALF-INTEGER RESONANCE $2 Q_{y}=9$}

\section{A. Results}

Measurements of beam intensity, transverse and longitudinal profiles evolution have been performed on a $160 \mathrm{MeV}$ plateau at a programmed (or bare) static working point of $(4.28,4.53)$, during the measurement window from $450 \mathrm{~ms}$ to $620 \mathrm{~ms}$. The programmed tune is defined as the one that is set in the control room through the nominal fields of the main quadrupoles in the machine, excluding eventual error contributions. To reach the initial conditions, the beam has been injected at $50 \mathrm{MeV}$ with a vertical tune below the half integer, then accelerated to $160 \mathrm{MeV}$. The working point has been raised above the $Q_{y}=4.5$ line by keeping the single normal quadrupolar corrector $\mathrm{ON}$ in order to minimize losses and beam deformation; measurements start at $450 \mathrm{~ms}$, as soon as the normal corrector is switched off. Two different longitudinal bunch settings with a double rf harmonics are considered: $8 \mathrm{kV}(h=1)+$ $8 \mathrm{kV}(h=2)$ in antiphase (long bunch), $8 \mathrm{kV}(h=1)+$ $8 \mathrm{kV}(h=2)$ in phase (short bunch). These settings, especially the long bunch at injection, are often used during the PSB operation, in order to minimize the SC tune spread. Table I shows the initial beam parameters. Figure 4 shows the measured and simulated intensity evolutions during the cycle time for the long bunch case. For this specific working point, at $620 \mathrm{~ms}$, the beam survival is $15 \%$ in case of the long bunch. Simulations have been set up including quadrupolar errors, which are necessary to excite the $2 Q_{y}=9$ resonance. The gradient errors, as described in Sec. II, are applied to the lattice and they shift the original working point $(4.28,4.53)$ toward the half-integer line $(4.285,4.517)$. The misalignment errors have also a contribution in the vertical detuning 
TABLE I. Half-integer case-measured initial beam parameters.

\begin{tabular}{lcc}
\hline \hline Initial beam parameters & Long bunch & Short bunch \\
\hline Bunch population $\left[10^{12} \mathrm{p}\right]$ & 1.39 & 1.32 \\
$\sigma_{x}, \sigma_{y}[\mathrm{~mm}]$ & $5.21,3.6$ & $6.05,3.67$ \\
$\epsilon_{x}, \epsilon_{y}[\mu \mathrm{mrad}]$ normalized $\mathrm{rms}$ & $2.64,2.05$ & $3.24,2.21$ \\
rf voltage $(h=1, h=2)[\mathrm{kV}]$ & 8,8 & 8,8 \\
rf cavities relative phase & $\pi$ & 0 \\
Total bunch length $[\mathrm{ns}]$ & 634 & 400 \\
Bunching factor & 0.44 & 0.24 \\
Momentum spread $(1 \sigma)$ & $1.35 \times 10^{-3}$ & $1.95 \times 10^{-3}$ \\
Programmed tune $\left[Q_{x}, Q_{y}\right]$ & $4.28,4.53$ & $4.28,4.53$ \\
Simulated SC tune shift $\left[\Delta Q_{x, y}\right]$ & $-0.16,-0.24$ & $-0.27,-0.37$ \\
\hline \hline
\end{tabular}

(4.284, 4.513), due to the introduction of slight skew component and longitudinal lattice changes, and in the losses distribution along the machine. The losses are simulated through a realistic set of apertures, which are distributed in the lattice components.

Table II shows the simulations settings that have been used in PTC-Orbit [5], the code selected for our spacecharge studies, which combines the tracking libraries of PTC with the particle-in-cell (PIC) modules of Orbit for collective effects (i.e., space charge) calculations. The distributions, that have been generated from the tracking, are bi-Gaussian in transverse and follow a reconstruction of the measured phase space in the longitudinal plane [6]. The number of SC nodes represents the number of locations in the lattice where the space charge forces are computed and applied through the PIC routines: 201 nodes mean, in average, one every $0.78 \mathrm{~m}$. The simulations are terminated when an apparent steady state situation occurs. One should note that all the ingredients need to be included in the simulations. As shown in Fig. 4 if no errors are included, space charge alone does not drive losses (blue line). If errors are included-but no SC, the losses are due only to chromaticity and saturate (brown line). Considering SC, if only quadrupolar errors-but no misalignments-are included, a qualitative agreement is achieved (solid magenta line). Then, if also the misalignment errors are considered, there is a significative improvement with also quantitative agreement (black line). The last improvement is similar to the results of simulation with realistic quadrupolar errors only and a slightly lower programmed vertical tune of $Q_{y}=4.525$ (dashed magenta line), with respect to the original $Q_{y}=4.53$. This decrement brings the effective tune down to $Q_{y}=4.512$, putting in evidence that the distance of the tune to the resonance line is fundamental, in this type of experiment. The effective tune is equal to the programmed tune plus the considered errors components. For these simulations, quadrupolar errors are the essential ingredient. Misalignment errors contribute to losses only through the vertical bare tune shift. If the same vertical tune shift is artificially included, which is
TABLE II. Simulation settings for the half-integer case.

\begin{tabular}{ll}
\hline \hline Longitudinal SC & ON $(128$ bins $)$ \\
Transverse SC & $2.5 \mathrm{D}$ PIC-FFT w/o \\
& boundaries \\
Number of bins [x, y] & 128,128 \\
Number of macrop. & $500 \times 10^{3}$ \\
Number of SC nodes & 201 \\
\hline \hline
\end{tabular}

equivalent with the tune shift due to the misalignment, the misalignment itself is no longer necessary.

The simulated and measured intensity curves agree both in the dynamic behavior and the final value, including the intensity level at which the slope changes.

The losses are evident in the longitudinal plane. Simulations agree with measurements (Fig. 5): at first, mainly larger longitudinal amplitude particles are lost, then the ones closer to the $+/-1$ rad longitudinal fixed points. This phenomenon is the equivalent of "bunch shortening" mechanism [7] for a double-harmonic longitudinal bucket, and will be discussed in Sec. III C. The beam size-both horizontal and vertical, stays similar over the $170 \mathrm{~ms}$.

The $1 \sigma$ Gaussian beam size stays the same along the simulation window and reflects the measurements. Figure 6 shows the transverse profiles measurements and simulations (referred to as PDF, particle density function) for the long bunch case, normalized with respect to the maximum values, where one can note that the $1 \sigma$ beam size and the Gaussian shapes are preserved. The asymmetric behavior of some of the profiles can be due to nonoptimal settings of the wire-scanners when big losses have already happened

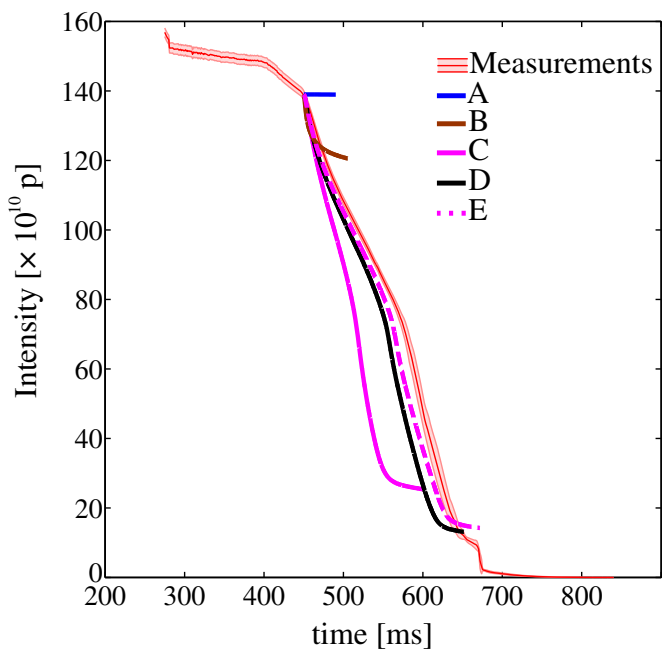

FIG. 4. Half-integer (long bunch simulations): PTC-Orbit simulations vs measurements. Legend: (A) Simulation without errors but with SC; (B) Simulation with errors but no SC; (C) Simulation with only quadrupolar field errors (matching to $Q_{y}=4.53$ ) and SC; (D) Simulation with SC and quadrupolar field and misalignment errors; (E) Simulation with only quadrupolar field errors (matching to $Q_{y}=4.525$ ) and SC. 

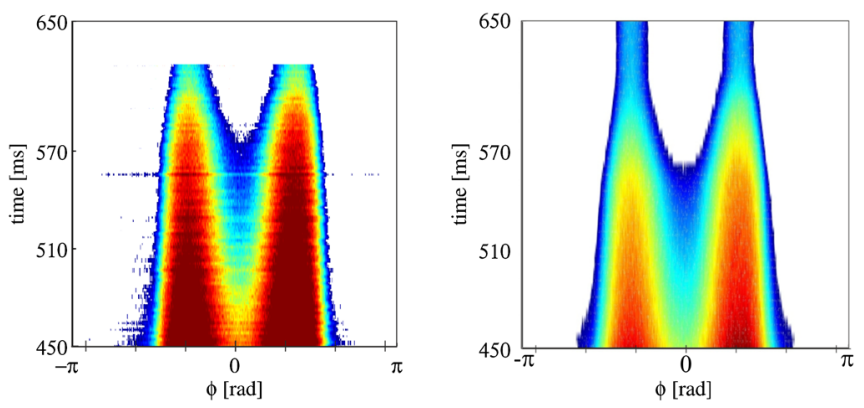

FIG. 5. Half-integer (long bunch): measured (left) and simulated (right) waterfall color plot in the time window [450-620] ms. The color code is the particle density (blue, low density—red, high density).

or to measurement limitations (i.e., scattering induced by the wire [8]). Simulations, moreover, revealed the formation of tails starting from $600 \mathrm{~ms}$. This effect was not seen in the measurements, most likely due to the noisy baseline in the wire-scanners profiles.

Very good qualitative and quantitative agreement is found also for the short-bunch case: Fig. 7 shows the measured $65 \%$ beam survival and the excellent matching in the intensity evolution between measurements and simulations. To compare the losses profiles for the same starting conditions, the simulated initial intensity and emittance for the short bunch have been put equal to the one of the long bunch (see Table I), even if they slightly differ in the measurements: this should only marginally affect the quality of the effects confirmed by the simulations in terms of profile shapes and quantities. The simulations include all
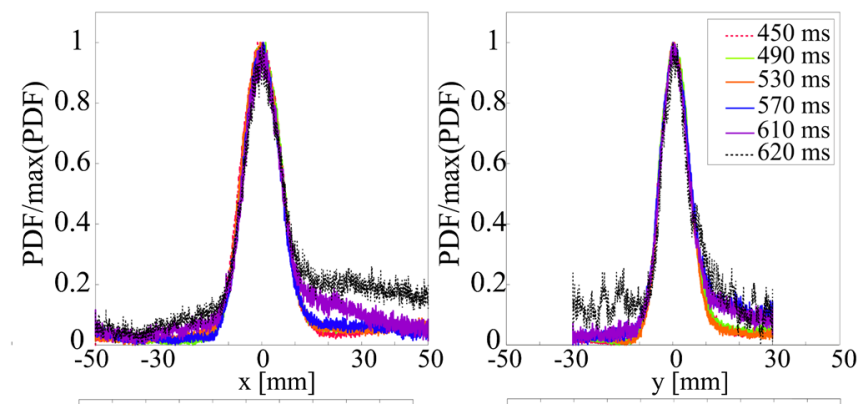

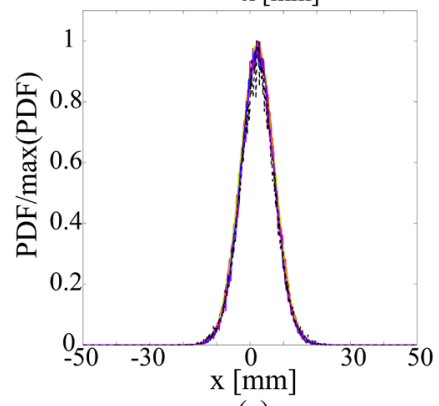

(a)

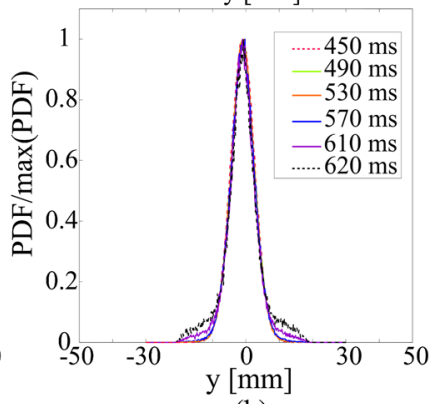

(b)
FIG. 6. Measured (top) and simulated (bottom) horizontal (a) and vertical (b) profiles in [450-620] $\mathrm{ms}$ for the long bunch case, normalized with respect to the max value.

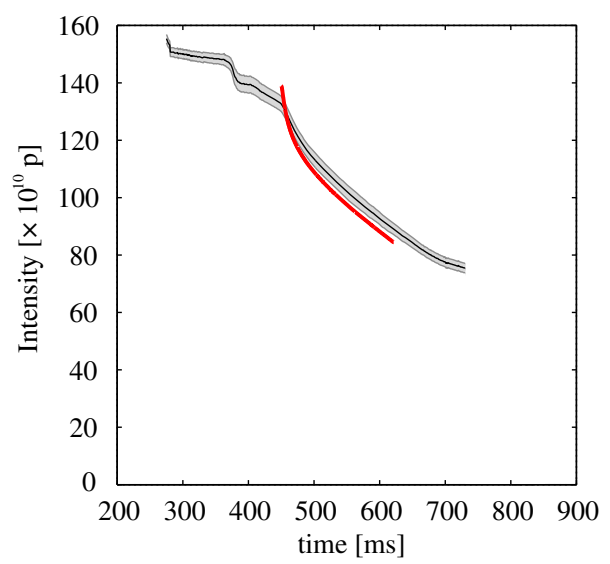

FIG. 7. Half-integer (short bunch). PTC-Orbit simulations vs measurements. Grey error bands are measurements, the red solid line are simulations with misalignment and quadrupolar field errors.

measured quadrupolar and misalignment errors, starting from a programmed tune of $(4.28,4.53)$, as in the previous case at the beginning, while Fig. 8 illustrates the bunch shortening effect taking place in the longitudinal plane.

\section{B. Effect of errors and working point}

Additional simulations to further explore the effect of errors and to identify what are the key parameters to take into account, in order, e.g., make prediction if the real set of machine errors is not known, are presented in Figs. 9 and 10.

Figure 9 shows, in addition to the lines in Fig. 4, the one matching the programmed tune at $Q_{y}=4.535$ (blue dashed line) plus only the realistic quadrupolar error applied and SC. A change of tune in the order of $10^{-2}$ causes a different final value in the intensity profile. Figure 9 shows also simulations with SC and 10 sets of random quadrupolar field errors (with different seeds), with the same standard
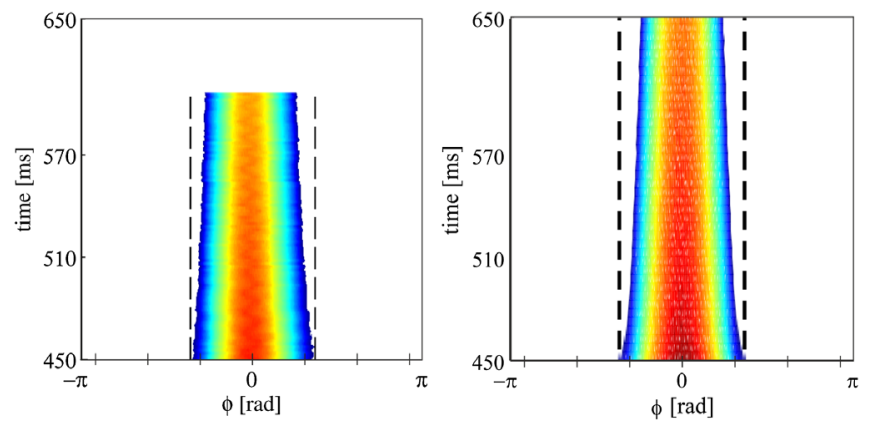

FIG. 8. Half-integer (short bunch): measured (left) and simulated (right) waterfall color plot in the time window [450605] ms. The color code is the particle density (blue, low density-red, high density). The comparison of the behavior with the vertical dashed black lines puts in evidence the bunch shortening. 


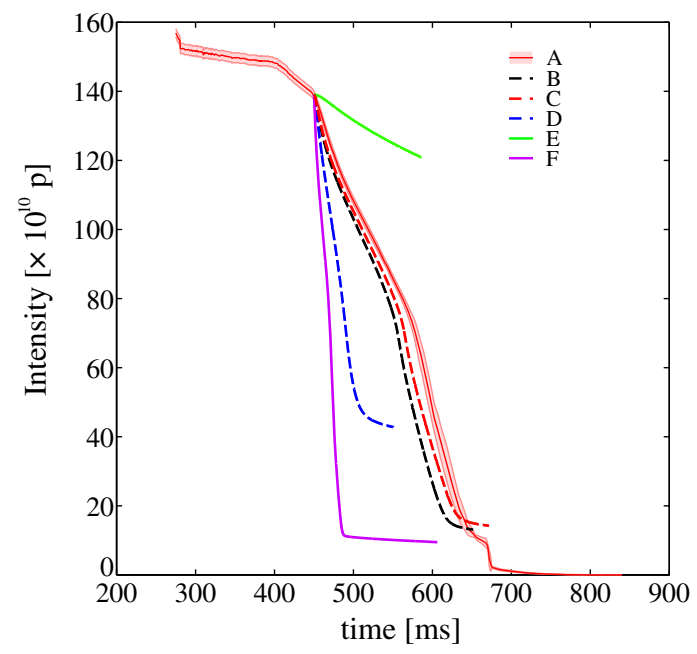

FIG. 9. Losses for different random errors. Legend: (A) Measurements; (B) Simulation with quadrupolar field and misalignment errors; (C) Simulation with only quadrupolar field errors (matching to $Q_{y}=4.525$ ); (D) Simulation with only quadrupolar field errors (matching to $Q_{y}=4.535$ ); (E) Simulation with random quadrupolar field errors and relative beta-beating = $3.46 \%$ rms; (F) Simulation with random quadrupolar field errors and relative beta-beating $=19.51 \% \mathrm{rms}$. All the simulations include SC.

deviation of the realistic ones, matched at the same final Twiss tune as in Fig. 4-E (4.2857, 4.512). The average effective tunes of these random-seed simulations are $Q_{x}=4.2856 \pm(5 \mathrm{e}-4)$ and $Q_{y}=4.5115 \pm(1.1 \mathrm{e}-3)$. From the losses profiles one can observe that, being the tunes so close, the behavior in time, including the intensity level at which the slopes change, and the final values are very similar.

The loss rates are different and depend on the beta-beating and, thus, on the resonance width [9]. Figure 10 shows the correlation between the vertical beta beating values and the initial loss rate, calculated as the (opposite) intensity derivatives when the curves are at $I=130 \times 10^{10} \mathrm{p}$, normalized with respect to the value of the slowest losses one (green curve of Fig. 9). For similar tunes, as in this case, the higher the beta beating, the faster the losses. This confirms in the simulations that beta-beating correction, possible in the machine through dedicated normal quadrupoles is effective for loss minimization.

\section{Discussion}

Simulations reproduce and explain losses, up to the detail of the change of slope for the long-bunch case, by correlating them to the specificity of the longitudinal motion in a double harmonic bucket in the presence of space-charge and in interaction with the $2 Q_{y}=9$ lattice resonance.

The bunch shortening process described, e.g., in [7] implies that particles at large synchrotron amplitude feel a

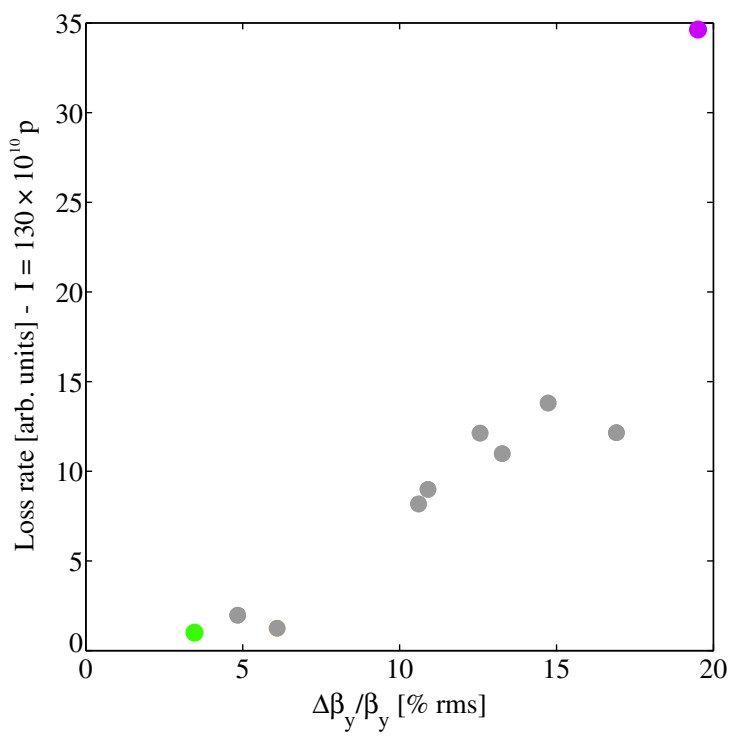

FIG. 10. Loss rate vs vertical beta beating at $130 \times 10^{10} \mathrm{p}$ for the different loss profiles. The curves are normalized with respect to the slowest loss rate. Colored points are the values of the loss rate for two different beta-beating seeds as shown in Fig. 9. The gray points are all the other simulated seeds.

large tune modulation induced by space-charge and they sample different bunch line densities during their synchrotron motion. Since the tune footprint overlaps the halfinteger line, these particles, which are periodically crossing the resonance line, are the candidates to be trapped or scattered and eventually to be lost. The phenomenon is evident in the longitudinal plane as a shortening of the bunch. Moreover, the loss of particles at large synchrotron amplitude has the consequence of reducing the spacecharge tune spread, therefore repopulating the regions affected by the interaction with the resonance and subjected to losses.

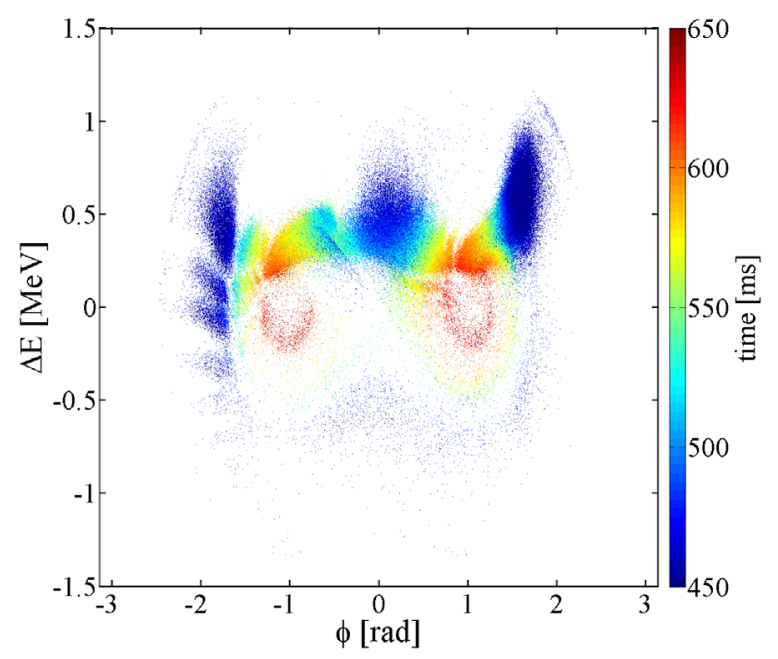

FIG. 11. Simulated position of the lost particles in the longitudinal phase space. The color code is the tracking time. 


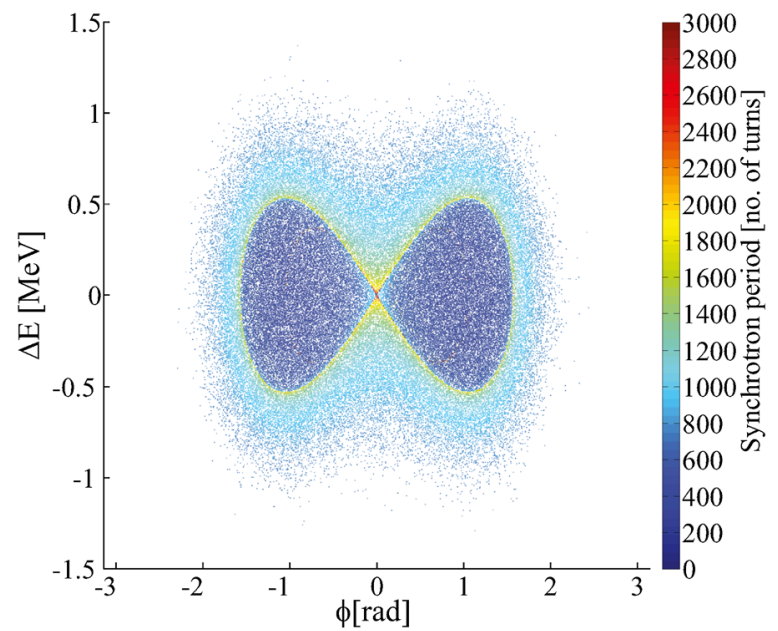

FIG. 12. The synchrotron period variation in a double harmonics rf bucket.

In a double-harmonics system, and in particular in our long-bunch case, the effect is similar, with the complication of having three fixed points at $\phi=0$ and $\phi \approx \pm 1 \mathrm{rad}$. An analysis of the longitudinal phase space coordinates of the lost particles, as shown in Fig. 11, demonstrates that the first particles to get lost are mainly the ones with large synchrotron amplitude.

A deeper analysis has been performed to correlate the losses with the synchrotron motion. Figure 12 puts in evidence the nonlinearity of the double harmonics rf bucket, showing the simulated variety of synchrotron periods for particles that belong to different iso-Hamiltonian trajectories in the longitudinal phase space. Particles rotating around the unstable point $\phi=0$ (which defines the inner separatrix) have a larger synchrotron period and cover bigger phase$\Delta p / p$ areas with respect to the ones that rotate in the limit cycles around $\pm 1 \mathrm{rad}$.

The synchrotron period variation has an effect on the loss rate. As soon as the particles outside the inner separatrix are

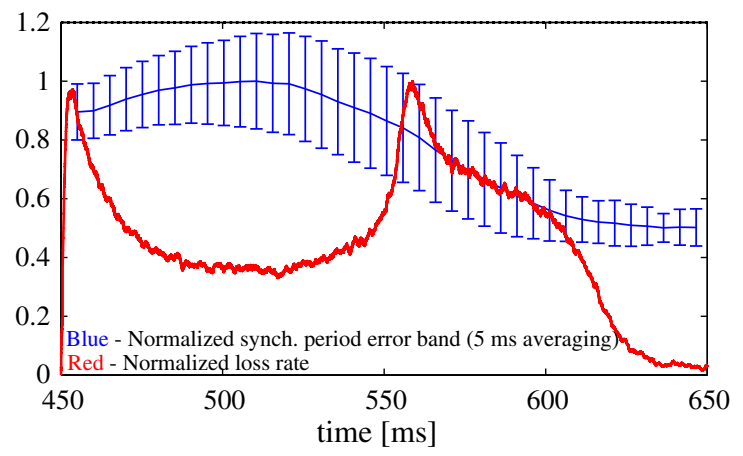

FIG. 13. Half-integer (long bunch simulations). The loss rate (red) vs the synchrotron period of the lost particles (blue). One can observe the different regimes before and after $558 \mathrm{~ms}$, where the bunch shortening starts to interest prevalently the particles inside the inner separatrix (with smaller synchrotron period).

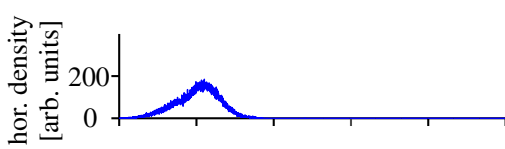

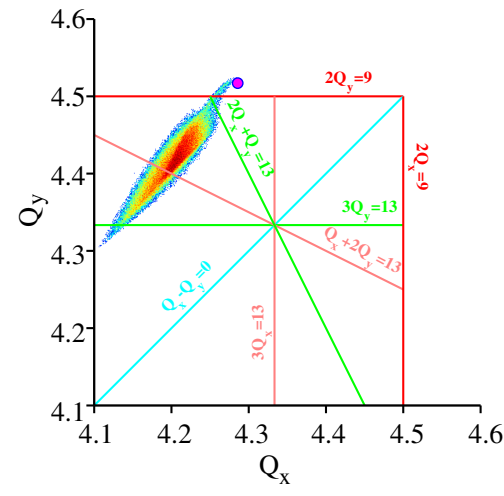

(a)
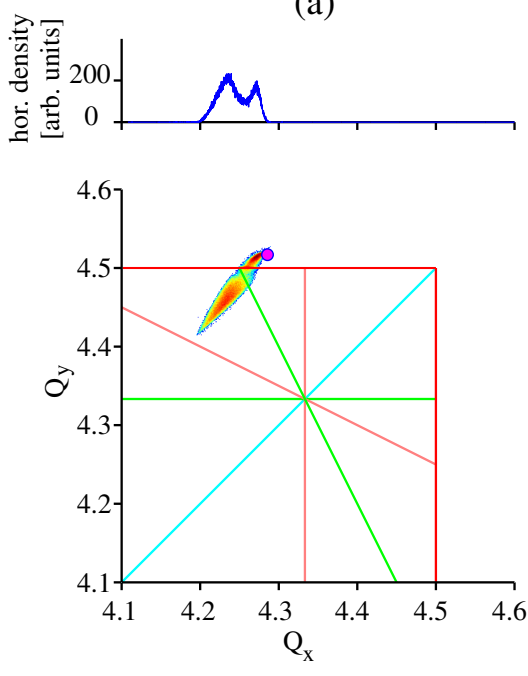

(b)

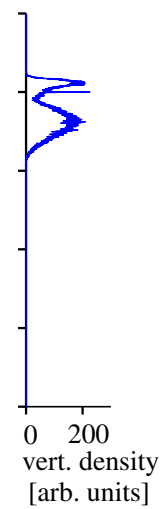

FIG. 14. The PTC-Orbit simulated tune footprint (averaged over 1500 turns) at $485 \mathrm{~ms}$ (a) and $565 \mathrm{~ms}$ (b), with particle density color code (blue, low density—red, high density). In (b), note the narrow peak on the $2 Q_{y}=9$ line.

lost, from $\sim 560 \mathrm{~ms}$, and the remaining ones, which oscillate around the $\pm 1 \mathrm{rad}$ equilibrium points, are the only ones to be affected (Figs. 5 right and 11), there is a change of slope in the intensity evolution. At this regard, one can see in Fig. 13 that losses are faster when the synchrotron period of the interested particles becomes smaller. Here, in red, one can see the loss rate along the cycle and, in blue, the average synchrotron period of the lost particles, sampled every $5 \mathrm{~ms}$. There are different regimes: at the very beginning a peak arises from the start of the simulations for $\sim 3 \mathrm{~ms}$. This regime can be considered as a transient as some synchrotron oscillations are needed for the space charge to develop the losses mechanism from the initial stationary regime. Then, before $558 \mathrm{~ms}$ particles are lost slower and in the interval [480-540] ms with an almost constant loss rate. These 


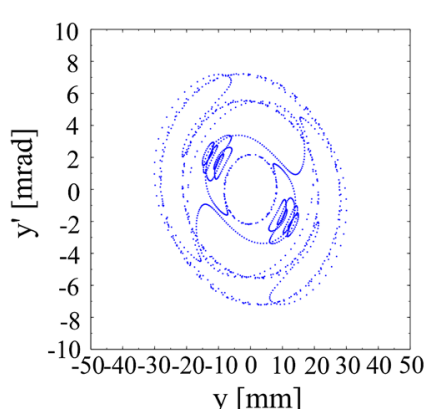

(a)

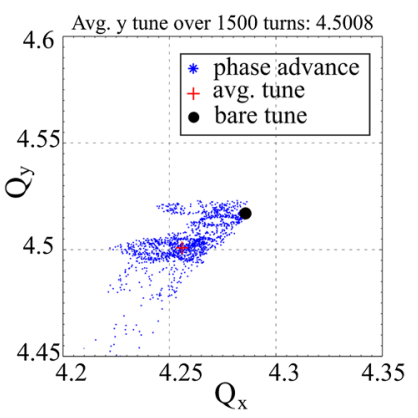

(b)
FIG. 15. A particle tracked at $485 \mathrm{~ms}$ [on the solid magenta line in Fig. 4(a)] before getting vertically lost by scattering. The turnby-turn tracking is represented with blue dots the vertical phase space (a) and in the tunes plane (b).

particles are the ones external to the inner separatix. When, after $558 \mathrm{~ms}$, as a consequence of the bunch shortening, the remaining particles are only the ones inside the basins of attraction around $\pm 1 \mathrm{rad}$ (Fig. 5 right), the loss rate increases for particles with smaller synchrotron period (the ones inside the inner separatrix).

Figure 14 shows the tune footprint at $485 \mathrm{~ms}$ (a) and at $565 \mathrm{~ms}$ (b), computed averaging the phase advance per turn over 1500 turns, as described in [10], for the simulations performed with quadrupolar fields errors only (magenta curve $\mathrm{C}$ of Fig. 4). In both plots, one can note the depletion along the half integer line. Moreover, the vertical projection of the averaged tune footprint, computed at $565 \mathrm{~ms}$, shows an interesting peak exactly at $Q_{y}=4.5$. A closer look at the Poincaré section in the $\left(y, y^{\prime}\right)$ plane of a particle, before it gets lost at around $485 \mathrm{~ms}$ (Fig. 15), puts in evidence the trappingscattering phenomenon [7], which leads the particles to eventually hit the vertical aperture. Figure 15(b) shows the turn by turn phase advance of the particle, and its averaged tune, which is sitting exactly on the vertical half integer.

A stable and steady-state condition occurs at $565 \mathrm{~ms}$, as soon as the losses are reduced to almost zero. In this case, the space charge forces are significantly smaller and the tune

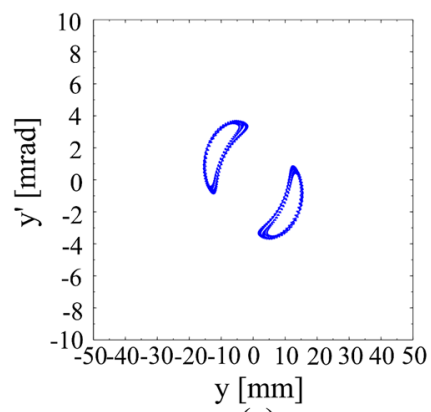

(a)

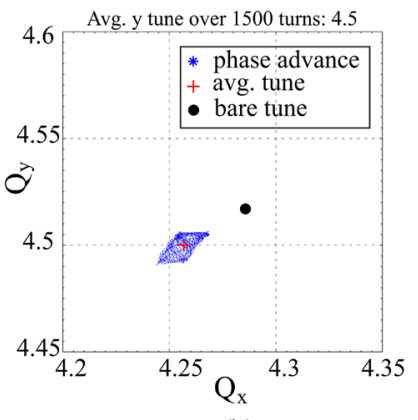

(b)
FIG. 16. A particle trapped into quadrupolar islands at $565 \mathrm{~ms}$ [on the solid magenta line in Fig. 4(a)]. The turn-by-turn tracking is represented with blue dots in the vertical phase space (a) and in the tunes plane (b). footprint [Fig. 14(b)] shows a clear peak on the $2 Q_{y}=9$ half-integer resonance line: particles that lie on this line are trapped into resonance islands (Fig 16) and create the halo in the vertical beam profile, as detected in the simulations, shown in Fig. 6.

\section{DYNAMIC APPROACH TO THE $Q_{y}=4$ RESONANCE}

The second case analyzed in this manuscript is the dynamic approach to the vertical integer resonance. Here the vertical tune is moved toward and then far from the resonance, while the horizontal is kept constant as shown in Fig. 17. The function of the programmed tune (Fig. 17) has been set up to have losses only for few milliseconds, in a way to preserve the measurability of the beam in the interval [500-570] ms. The programmed tunes are the nominal ones, which differ from the measured (coherent) ones due to the effect of the quadrupolar and misalignment errors and the intensity-dependence of the coherent tune.

The dipolar errors of the machine are calibrated in the simulation by applying additional kicks through 13 available vertical orbit correctors (DVTs), as explained in Sec. II, to get the same COD as in the measurements. On top of this calibration, some of these DVTs can be powered with an extra current to take into account the vertical steering correction performed in the machine [11]. Simulations have been performed and compared with measurements including or not this last COD correction.

Table III shows the initial beam parameters, referring to a short bunch, and Table IV the initial simulation settings. The quantities for the corrected-COD analysis have been chosen as starting beam parameters. One has to say that the calibration of the COD has not been readjusted for each quadrupolar error seed.

\section{A. Results without COD correction}

Figure 18 reports the measured intensity evolution in case the COD correction is not applied (blue solid line) together with simulations.

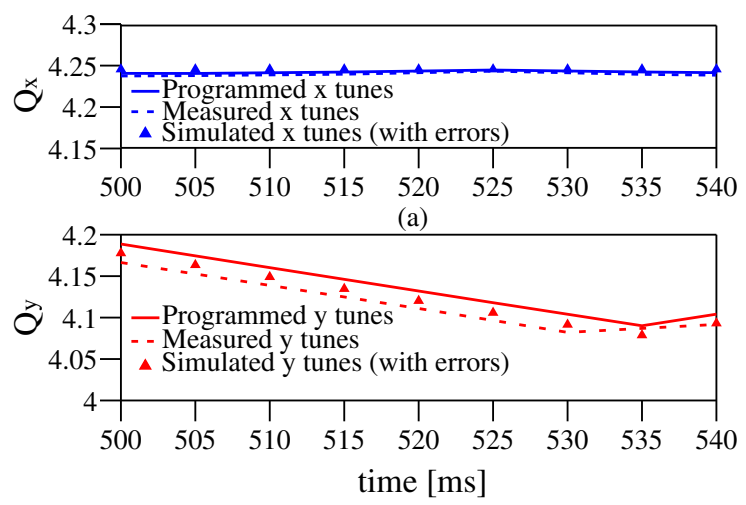

(b)

FIG. 17. The dynamic approach to $Q_{y}=4$ : horizontal (a) and vertical (b) tunes vs time. 
TABLE III. Measured initial parameters for the dynamic integer case.

\begin{tabular}{lc}
\hline \hline Initial beam parameters & Short bunch \\
\hline Bunch population $\left[10^{12} \mathrm{p}\right]$ & 1.7 \\
$\sigma_{x}, \sigma_{y}[\mathrm{~mm}]$ & $5.68,5.96$ \\
$\epsilon_{x}, \epsilon_{y}[\mu \mathrm{mrad}]$ normalized rms & $2.68,5.05$ \\
rf voltage $(h=1, h=2)[\mathrm{kV}]$ & 8,8 \\
rf cavities relative phase & 0 \\
Total bunch length $[\mathrm{ns}]$ & 400 \\
Bunching factor & 0.24 \\
Momentum spread $(1 \sigma)$ & $1.95 \times 10^{-3}$ \\
Tune $\left[Q_{x}, Q_{y}\right]$ & $4.24,4.19$ \\
Simulated SC tune shift $\left[\Delta Q_{x, y}\right]$ & $-0.31,-0.24$ \\
\hline \hline
\end{tabular}

The tracking without space charge (purple dashed curve) shows no losses, while the simulation including the direct space charge and all the measured (misalignments plus quadrupolar) errors shows an exceptional overlap with the measurements. Moreover, to confirm that the correct quadrupolar field errors are important for these simulations, a set of normally distributed random errors has been generated for the quadrupolar fields (focusing and defocusing), with the same standard deviation of the real errors $\left(\sigma(\delta K 1)=0.7 \times 10^{-3}\right)$. This, again, generates different beta-beatings and, thus, different resonance widths [9]. In the case of random errors, simulations do not show good agreement (pink error bar) and neither if field errors are not considered, i.e., only misalignments and dipole errors in the steerers are (green continuous line). A big contribution to the losses is due to the COD, which increases as the beam approaches the integer resonance. It was not possible to measure the transverse profiles through the wire-scanners in this situation, due to large losses.

Figure 19 shows the simulated beam profiles: in case the complete error set is applied and the space charge calculation is OFF, the beam only shifts due to the COD blow-up (a), while when space charge is $\mathrm{ON}$, there is also an emittance blow-up (b), as expected in proximity of the integer resonance. Differently from the previous case,

TABLE IV. Simulation settings for the dynamic integer case (with and without boundaries).

\begin{tabular}{ll}
\hline Number of macroparticles & $500 \times 10^{3}$ \\
Number of SC nodes & 201 \\
Longitudinal SC & ON $(128$ bins $)$ \\
Transverse SC model & $\begin{array}{l}2.5 D \text { PIC-FFT without } \\
\text { boundaries }\end{array}$ \\
Number of bins [x, y] & 128,128 \\
Transverse SC model & $2.5 D$ PIC-FFT with boundaries \\
Chamber type & Rectangular \\
Chamber size & $\pm 61 \mathrm{~mm} \times \pm 29.5 \mathrm{~mm}( \pm 40 \mathrm{~mm})$ \\
Number of bins $[\mathrm{x}, \mathrm{y}]$ & 256,256 \\
\hline \hline
\end{tabular}

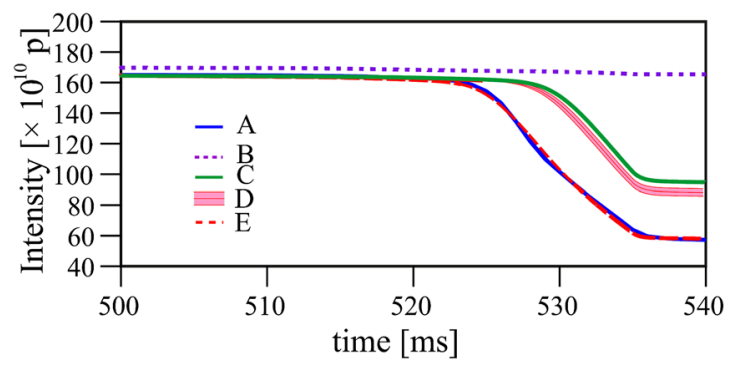

FIG. 18. The dynamic approach to $Q_{y}=4$ : intensities vs time without COD correction. Legend: (A) Measurements; (B) Simulation without space charge; (C) Simulation with direct space charge; (D) Simulations error bar with direct space charge and random quadrupolar field errors distributions; (E) Simulation with measured quadrupolar fields errors and direct space charge.

where the bare tune was very close to the half-integer resonance and the particles had continuous interaction with the half-integer line, being lost after some time, now the tune spread touches the resonance with its lower part. This perturbs the motion of the particles in contact with the resonance and changes their actions. Core particles are affected by scattering and the interactions with the resonance line stops when their amplitude is increased enough, inducing lower space charge tune shifts. This effect, applied to many particles, induces the overall blow-up.

\section{B. Results with COD correction}

Simulations have been performed also for the case with the COD correction (measurements are the blue solid line in Fig. 20), in order to disentangle the space charge effects from the COD ones. As before, if space charge is not included in the calculations, the behavior of the intensity is pretty flat (black dashed line), while in case space-charge is added to the tracking, losses occur: the simulation with direct space charge (red dashed curve) underestimates the losses, while the one using the PTC-Orbit module including the boundaries with the smallest vertical aperture

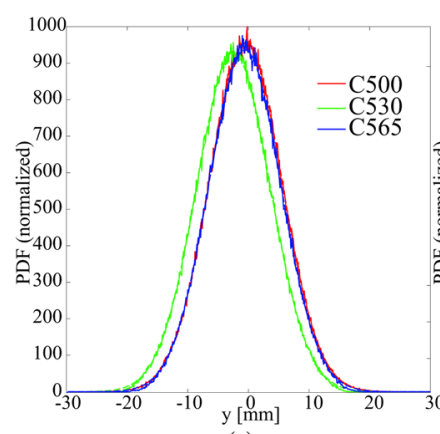

(a)

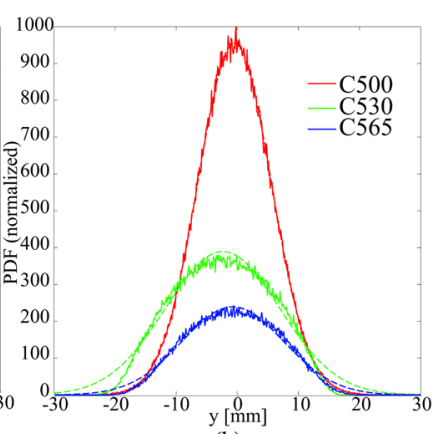

(b)
FIG. 19. The simulated vertical profiles with uncorrected COD, without (a) and with space charge (b). The dashed lines are the Gaussian fits. 


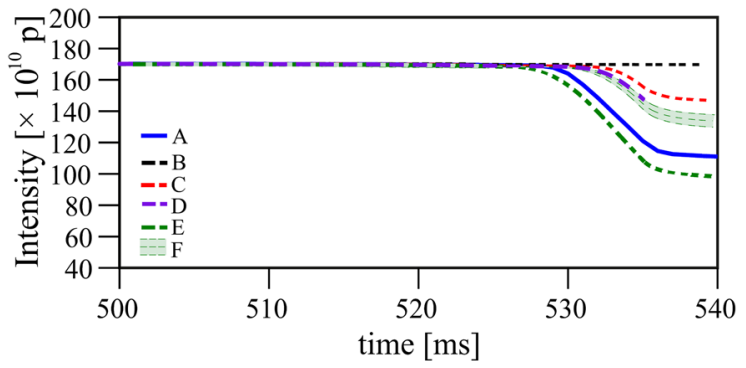

FIG. 20. The dynamic approach to $Q_{y}=4$ in case of corrected COD: intensities vs time with vertical boundaries at $\pm 29.5 \mathrm{~mm}$. Legend: (A) Measurement; (B) Simulation without space charge; (C) Simulation with direct space charge; (D) Simulation with space charge (with boundaries) without field errors; (E) Simulation with space charge (with boundaries) and measured quadrupolar field errors; (F) Simulation with space charge (with boundaries) and random quadrupolar field errors distributions.

(rectangular with dimensions $\pm 61 \mathrm{~mm} \times \pm 29.5 \mathrm{~mm}$ ), slightly overestimates them (dashed green line).

Different error distributions generate different intensity results: the green error bar represents the simulations behaviour in case a random error set of quadrupolar field errors is chosen, while the simple dashed green line is a similar simulation, but using the errors as they have been calculated on the real machine through the LOCO analysis. The dashed purple line represents the intensity profile obtained in case no field errors are considered.

In this case, vertical wire-scanner measurements were possible and are reported in Fig. 21(a). Figure 21(b) shows simulations including direct and indirect space charge. As in the previous case, without COD correction, the beam blow-up is clear and fast and the beam becomes nonGaussian already after $1 \mathrm{~ms}$ (Fig. 22). The rms emittance behavior is shown in Fig. 23: the vertical measurements

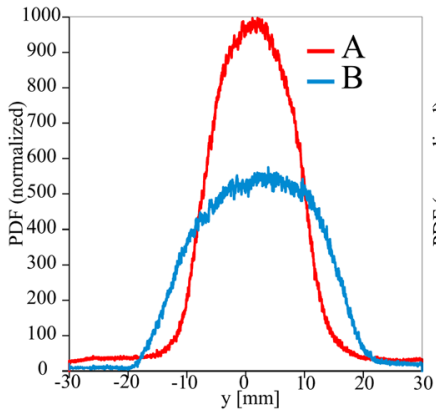

(a)

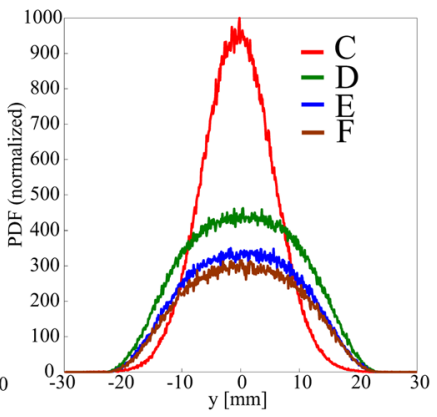

(b)
FIG. 21. The vertical profiles in case of COD correction: (a) Measurements, (b) Simulations including the direct space charge and the boundaries. Legend-left: (A) Measurements at $500 \mathrm{~ms}$; (B) Measurements at $560 \mathrm{~ms}$. Legend-right: (C) Simulation: initial vertical profile at $500 \mathrm{~ms}$; (D) Vertical profile at $560 \mathrm{~ms}$ (with direct space charge); (E) Vertical profile at $560 \mathrm{~ms}$ (SC with vertical boundaries at $\pm 40 \mathrm{~mm}$ ); (F) Vertical profile at $560 \mathrm{~ms}$ ( $\mathrm{SC}$ with vertical boundaries at $\pm 29.5 \mathrm{~mm}$ ).

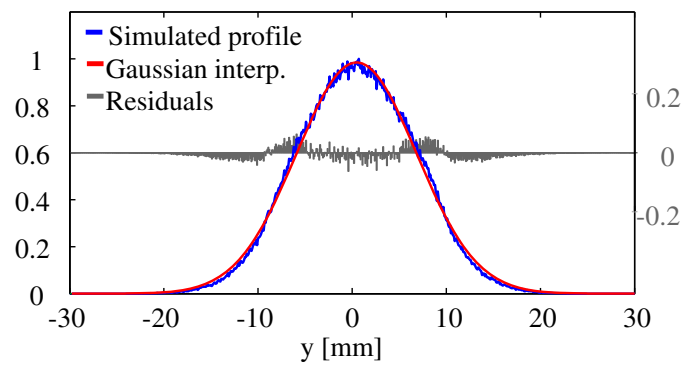

FIG. 22. The Gaussian shape is lost already after 1000 turns (1 ms).

(Gaussian fit of the wire-scanner profiles) are the pink error bar, while the simulations are dashed in case of statistical computation [12], or represented with markers in case of simulated Gaussian fit of the vertical beam profile, as it is commonly performed in the PSB control room. The discrepancy between the statistical and the Gaussian fits clearly demonstrates the loss of Gaussianity due to the lower part of the tune spread approaching the integer resonance. One must say that the initial measured distribution is not completely Gaussian, while in the simulations a Gaussian starting profile has been used in every case. This leads to small errors in the computation of the initial space charge tune spreads.
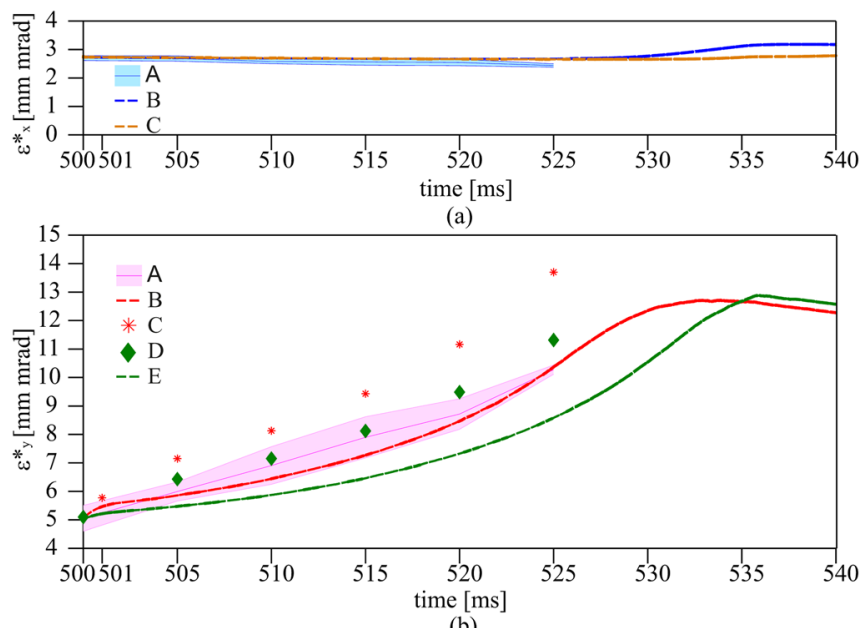

(b)

FIG. 23. The dynamic approach to $Q_{y}=4$ : the normalized horizontal (a) and vertical (b) rms emittances behavior for the space charge simulations including and excluding the boundaries ( $\pm 61 \mathrm{~mm} \times \pm 29.5 \mathrm{~mm}$ ). The simulations represent the case with the vertical orbit correctors ON. Legend-top: (A) Measurements (Gaussian fit of wire-scanner profiles); (B) Simulated statistical emittance (with SC with boundaries); (C) Simulated statistical emittance (direct space charge). Legend-bottom: (A) Measurements (Gaussian fit of wire-scanner profiles); (B) Simulated statistical emittance (with SC with boundaries); (C) Simulated emittance from Gaussian fit (with SC with boundaries); (D) Simulated emittance from Gaussian fit (with direct SC); (E) Simulated statistical emittance (with direct SC). 


\section{Calibration of the boundary conditions}

The PTC-Orbit code permits the definition of only a single set of boundary conditions of a defined shape (circular, rectangular, elliptical), considering it equal all around the machine. An accelerator has usually different vacuum chamber sizes and shapes along the machine, so an "equivalent" one has to be defined for the tracking with transverse indirect space charge. The method here used intends to find the conditions for which the simulations overlap the measurements in the case with COD correction, in which the beam deformation is clearly dominated by space charge. For this reason different simulations have been launched with different heights for a rectangular shape, having fixed the horizontal one to $\pm 61 \mathrm{~mm}$.

Figure 24 shows the simulation with different heights of the vacuum pipe. In this case the $\pm 40 \mathrm{~mm}$ solution is the most suitable in comparison with the measurements. This indeed reflects an average vertical aperture size of the machine, while the $\pm 29.5 \mathrm{~mm}$ corresponds to the minimum distributed vertical aperture, which is defined by the scrapers before and after each bending magnet.

Figure 25 shows the effect of the different shape boundaries on the coherent tunes. In these plots the coherent tunes are computed through fast Fourier transform (FFT) of the first order moments along the simulation window. In case of small boundaries $( \pm 29.5 \mathrm{~mm})$ the FFT is more broadband and the vertical tune is closer to the integer. When the boundaries become larger, the vertical coherent tunes are slightly farther from the resonance and more narrow-band, with higher peaks in frequency as one moves toward the direct space charge condition (and also for very large chambers), as shown in Fig. 26. The tune shift effect can be also correlated to what happens in the case without COD correction: Figure 27 shows different simulation results for different chambers size. Here, as already shown, the divergence of the COD influences sensibly the losses pattern: a different coherent tune shift toward the vertical integer causes an initial mismatch of the

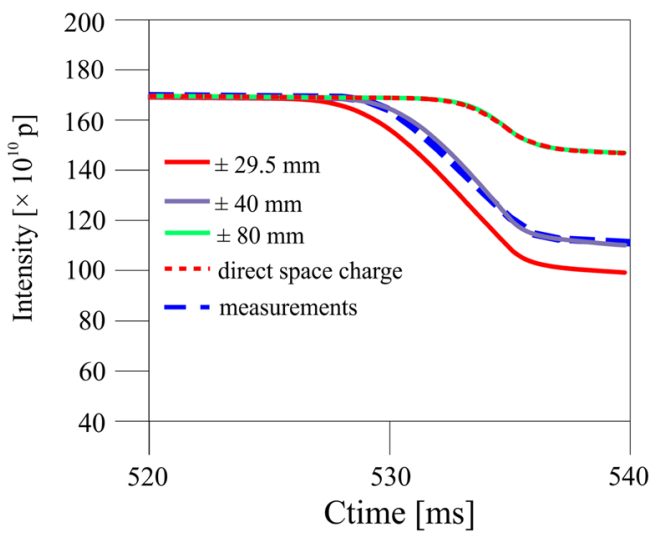

FIG. 24. Dynamic integer with COD correction: simulations with different chamber heights.
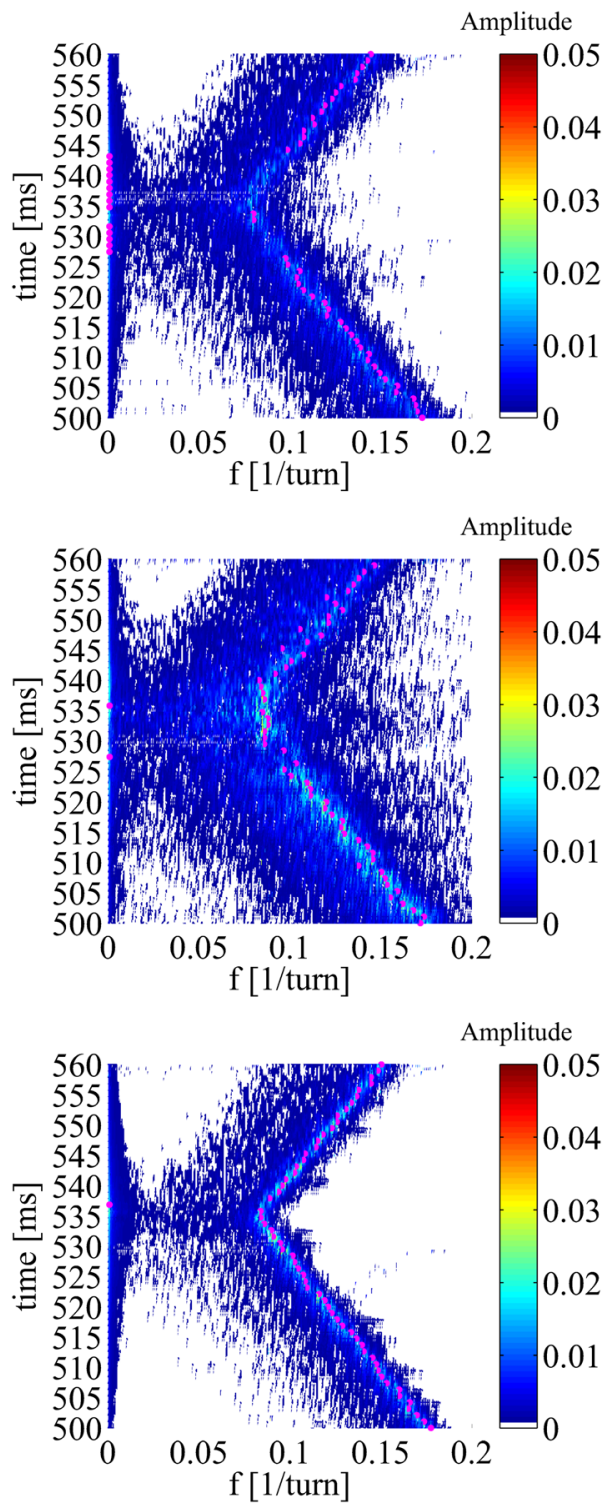

FIG. 25. Vertical coherent tunes simulations with COD correction. From bottom to top the following space charge modules have been used: vertical boundaries $\pm 40 \mathrm{~mm}$; vertical boundaries $\pm 29.5 \mathrm{~mm}$; direct space charge. The magenta dots represent the peaks in the FFT of the first order momenta.

COD, that, feeling a dipolar blow-up along the tune path, anticipates or delays the losses.

\section{Discussion}

The vertical integer resonance $Q_{y}=4$ has been approached dynamically: the coupled effect of space charge and closed orbit distortion (COD) on beam losses has been observed and reproduced with simulations. This case has exhibited both rms and COD blow-up during the procedure. Without the COD correction the simulation results have been excellent, showing high sensitivity to the COD induced by the realistic machine errors. 


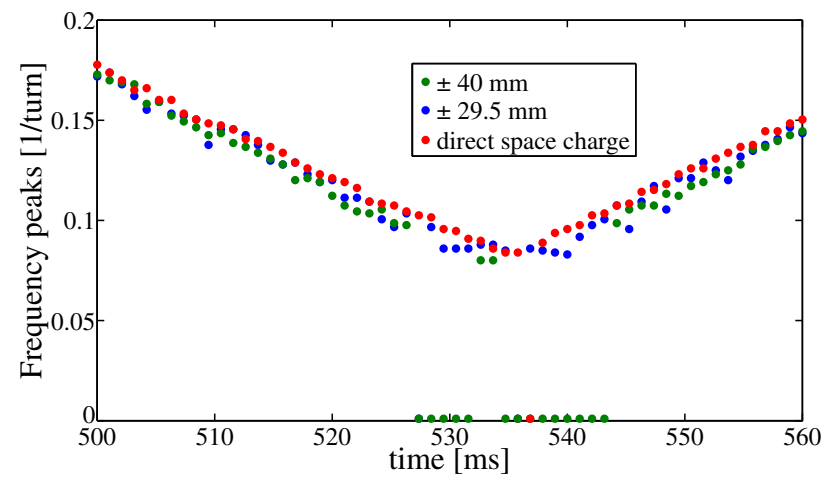

FIG. 26. Vertical coherent tune peaks. The dots are the maxima of the first order vertical momenta of the beam (magenta points of Fig. 25).

On the other hand, with the COD correction, the direct space charge simulation model did not fully describe the measurements. The PTC-Orbit model including the boundaries for the indirect space charge computation has been also adopted, as the beam became very large during the blow-up induced by the integer resonance. Moreover, an improvement of the model has been reached by a simulated equivalent chamber calibration, that has been performed to
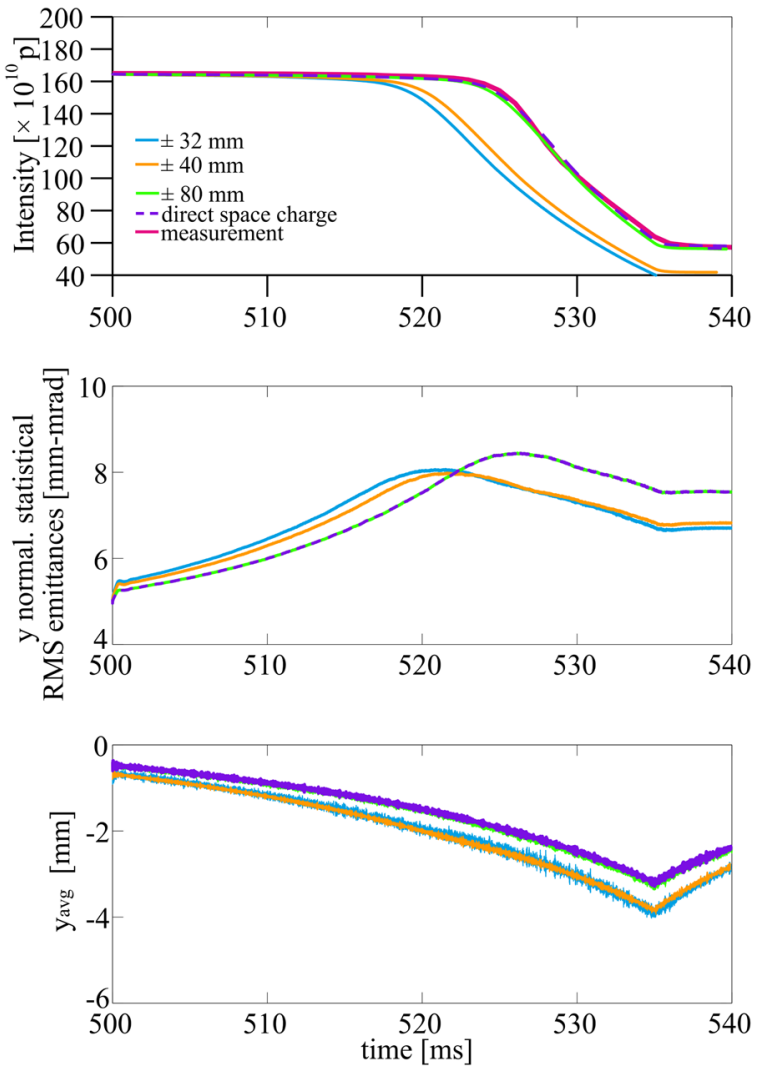

FIG. 27. Dynamic integer without COD correction: simulations with different chamber heights of intensity (top), normalized statistical emittance (center) and vertical centroid (bottom). retrieve an optimal equivalent distance in the vertical plane. The calibration has shown good results for the case with COD correction. However, in the case without COD correction, the presence of a coherent tune shift, caused by the indirect space charge component, induced a deviation of the closed orbit from the beginning, with respect to the measured one. This had the effect of anticipating the losses for smaller chamber sizes, causing a disagreement. A rematching of the COD, compensating the indirect space charge induced variation, may improve the results.

\section{CONCLUSIONS}

The combined effect of a realistic set of errors (misalignments and quadrupolar field errors) and space charge has been analyzed in a benchmarking between measurements and simulations with the PTC-Orbit code in the PSB. Two cases have been taken into account.

In the first case, with static tunes close to the $2 Q_{y}=9$ half-integer resonance, simulations have shown very good agreement with the measurements for long and short bunches. The results can be explained by the bunch shortening phenomenon due to the trapping-scattering mechanism proposed by Franchetti et al. [7]. Selfconsistency of the model is important and PIC simulations (with the PTC-Orbit code) are needed to correctly model the case of a double-harmonic nonlinear bucket. The losses depend on the tunes, on the distance from the half-integer line and on the beta-beating. Having identified these two ingredients is essential in order to make predictions for the future beams after the LIU Upgrade. We are planning further experiments at the PSB where we will vary the excitation of the resonance line modulating the strength of the normal quadrupolar corrector, which has also been used in this paper. This will allow us to partially correct the resonance and arbitrarily vary the losses up to a few percent. Varying the corrector, a systematic benchmarking with simulations will then be possible.

In the second case, where the integer resonance $Q_{y}=4$ is being approached, the coupled effect of space charge and closed orbit distortion (COD) on beam losses has been demonstrated. This case exhibits both rms beam size and COD blow-up during the procedure. Without the COD correction the simulation results are excellent in the presence of direct space charge and closed orbit calibration. On the other hand, with the COD correction, our simulation model does not yet fully describe the measurements. An improvement of the model has been reached introducing the (indirect) contribution of image effects. In this case, in fact, the closed orbit does not blow-up as previously, but the beam size reaches such high values that image effects can not be neglected any longer. An equivalent chamber calibration has been also performed in simulations to retrieve an optimal equivalent distance in the vertical plane. 


\section{ACKNOWLEDGMENTS}

The authors would like to warmly thank all the PSB-OP team for the determinant support during the measurements, A. Yu. Molodozhentsev (KEK, Japan), G. Franchetti (GSI, Dramstadt), C. Carli, and F. Schmidt (CERN) for the numerical and theoretical hints. The authors would like to thank the CERN LHC injectors upgrade (LIU) project and the BE-ABP group for funding the research work related to this article.

[1] J. Safranek, Experimental determination of storage ring optics using orbit response measurements, Nucl. Instrum. Methods Phys. Res, Sect. A 388, 27 (1997).

[2] M. McAteer, Ph.D. thesis, University of Texas, 2014.

[3] T. Dobers (private communication).
[4] M. McAteer et al., Linear optics from orbit response measurements in the PS Booster, CERN-ATS (to be published).

[5] A. Shishlo et al., KEK Internal Report (A), Report No. 2007-4, 2007.

[6] S. Hancock (private communication).

[7] G. Franchetti et al., Experiment on space charge driven nonlinear resonance crossing in an ion synchrotron, Phys. Rev. Accel. Beams 13, 114203 (2010).

[8] F. Roncarolo, Ph.D. thesis, EPFL Lausanne, 2005; Report No. CERN-THESIS-2005-082.

[9] H. Wiedemann, Particle Accelerator Physics (Springer, New York, 2015).

[10] V. Forte et al., 6D Tunes Computation For Space Charge Dominated Beams (to be published).

[11] V. Forte et al., MD Report No. CERN-ACC-NOTE-20140056, 2014.

[12] J. Buon, Reports No. LAL-RF-90-15-REV, No. LAL-RT-9203; CAS-CERN Accelerator School: 5th General Accelerator Physics Course (Jyvaskyla, Finland, 1992), pp. 89-116. 